\title{
SESAMs for High-Power Oscillators: Design Guidelines and Damage Thresholds
}

\author{
Clara J. Saraceno, Cinia Schriber, Mario Mangold, Martin Hoffmann, Oliver H. Heckl, Cyrill R. E. Baer, \\ Matthias Golling, Thomas Südmeyer, and Ursula Keller
}

(Invited Paper)

\begin{abstract}
We present for the first time to the best of our knowledge a systematic study of lifetime and damage of semiconductor saturable absorber mirrors (SESAMs) designed for operation in high-power oscillators. We characterize and compare nonlinear reflectivity and inverse saturable absorption (ISA) parameters as well as damage threshold and lifetime of different representative SESAMs under test using a nonlinear reflectivity measurement setup at unprecedented high fluence levels. We investigate the catastrophic damage that occurs at very high fluences by demonstrating a dependence of the damage threshold on the ISA parameter $F_{2}$ and the maximum reflectivity fluence $F_{0}$. We can clearly demonstrate that the damage fluence $F_{d}$ scales proportionally to $\sqrt{F_{2}}$ for all SESAMs. In the case of SESAMs with the same absorber where the product $F_{\text {sat }} \cdot \Delta R$ is constant, the damage fluence $F_{d}$ scales proportionally to $F_{0}$. Therefore, damage occurs due to heating of the lattice by the energy absorbed due to the ISA process and is not related to the quantum well (QW) absorbers. Furthermore, we present guidelines on how to design samples with high saturation fluences, reduced induced absorption, and high damage thresholds. Using multiple QWs and a suitable dielectric topsection, we achieved SESAMs with saturation fluences $>200 \mu \mathrm{J} / \mathrm{cm}^{2}$, nonsaturable losses $<0.1 \%$, and reduced ISA. Our best sample could not be damaged at a maximum available fluence of $0.21 \mathrm{~J} / \mathrm{cm}^{2}$ and a peak intensity of $370 \mathrm{GW} / \mathrm{cm}^{2}$. These SESAMs will be suitable for future high-power femtosecond oscillators in the kilowatt average output power regime, which is very interesting for attosecond science and industrial material processing applications.
\end{abstract}

Index Terms-High-power lasers, modelocked lasers, semiconductor saturable absorber mirrors (SESAMs), thin-disk lasers.

\section{INTRODUCTION}

$\mathbf{F}$ EMTOSECOND and picosecond lasers have progressed from complicated and specialized laboratory systems to widely used scientific and industrial instruments [1], [2]. Their ultrashort pulse durations, high peak powers, and unique spectral properties have resulted in numerous scientific discoveries and a growing number of applications in

Manuscript received September 7, 2010; revised November 9, 2010; accepted November 9, 2010. Date of publication January 13, 2011; date of current version January 31, 2012. This work was supported by the Swiss National Science Foundation.

The authors are with the Institute for Quantum Electronics, Eidgenössische Technische Hochschule Zürich, 8093 Zurich, Switzerland (e-mail: saraceno@phys.ethz.ch; scinia@student.ethz.ch; mangoldm@phys.ethz.ch; mh@phys.ethz.ch; heckl@phys.ethz.ch; cbaer@phys.ethz.ch; golling@first. ethz.ch; sudmeyer@phys.ethz.ch; keller@phys.ethz.ch).

Color versions of one or more of the figures in this paper are available online at http://ieeexplore.ieee.org.

Digital Object Identifier 10.1109/JSTQE.2010.2092753 various fields, such as physics, chemistry, biology, materials science, imaging, material processing, communication, and medicine. The invention of the semiconductor saturable absorber mirror (SESAM) [3], [4] nearly 20 years ago was a major advancement for the development of simple and reliable ultrafast laser systems. It enabled the first stable and self-starting passive modelocking of diode-pumped solid-state lasers (DPSSLs), resolving the long-standing Q-switching problem [5]. Today, SESAMs have become key devices for modelocking of numerous laser types, including DPSSLs, fiber lasers, and semiconductor lasers. Semiconductors are ideally suited as saturable absorbers because they can cover a broad wavelength range and yield short recovery times, supporting the generation of picosecond to femtosecond pulse durations. The macroscopic nonlinear optical parameters for modelocking can be optimized over a wide range by the design of the mirror structure and the choice of the semiconductor absorber

SESAM modelocking is currently the best-suited technology for high-power ultrafast laser oscillators. Recent SESAM modelocked thin-disk lasers have achieved average powers $>140 \mathrm{~W}$ [6] and pulse energies $>25 \mu \mathrm{J}$ [7], which are higher than for any other ultrafast oscillator technology. The combination of high power levels and multimegahertz repetition rates makes these lasers highly attractive for areas such as high-field laser science or industrial high-speed material processing [8], which were previously restricted to complex amplifier systems. Initial photoionization studies in noble gases clearly showed the advantages of driving high field science experiments at multimegahertz repetition rate, such as reduced measurement time, high signal-to-noise ratio (SNR), and elimination of space charge issues [8]. Driving high harmonic generation [9], [10] by high-power oscillators opens up new possibilities for increasing the average VUV/XUV photon flux by the higher average power of the driving laser [11], possibly in combination with enhanced phase matching in microstructured waveguides (e.g., hollow core photonic crystal fibers (PCF) [12]). Such compact megahertz sources of coherent radiation in the VUV/XUV spectral region with possibly even attosecond durations would have high impact on numerous areas such as medicine, biology, chemistry, physics, or material science.

The thin-disk laser concept [13], [14] offers excellent heat removal capabilities, that combined with SESAM modelocking represents a power scalable ultrafast technology [8]. The output power of the oscillator can be increased simply by increasing the pump power with both an increased pump spot size on the disk and laser spot size on the SESAM by the same factor. In 
TABLE I

TyPical OPERATION PARAMETERS OF SESAMs Used IN HIgH-POWER THIN-Disk LASERS

\begin{tabular}{|c|c|c|c|}
\hline Symbol & Yb:YAG [15] & Yb:YAG [7] & $\mathrm{Yb}: \mathrm{Lu}_{2} \mathrm{O}_{3}[6]$ \\
\hline Average output power & $45 \mathrm{~W}$ & $76 \mathrm{~W}$ & $140 \mathrm{~W}$ \\
\hline Repetition rate & $4 \mathrm{MHz}$ & $2.93 \mathrm{MHz}$ & $60 \mathrm{MHz}$ \\
\hline Intra-cavity average power & $450 \mathrm{~W}$ & $97.4 \mathrm{~W}$ & $1.49 \mathrm{~kW}$ \\
\hline Intra-cavity peak power & $125.7 \mathrm{MW}$ & $31.5 \mathrm{MW}$ & $29.8 \mathrm{MW}$ \\
\hline Output pulse energy & $11.3 \mu \mathrm{J}$ & $25.9 \mu \mathrm{J}$ & $2.35 \mu \mathrm{J}$ \\
\hline Intra-cavity pulse energy & $113 \mu \mathrm{J}$ & $33.2 \mu \mathrm{J}$ & $25 \mu \mathrm{J}$ \\
\hline Fluence on SESAM & $\approx 5-7 \mathrm{~mJ} / \mathrm{cm}^{2}$ & $\approx 4 \mathrm{~mJ} / \mathrm{cm}^{2}$ & $\approx 2 \mathrm{~mJ} / \mathrm{cm}^{2}$ \\
\hline Average intensity on SESAM & $\approx 2.9 \mathrm{~kW} / \mathrm{cm}^{2}$ & $\approx 1.5 \mathrm{~kW} / \mathrm{cm}^{2}$ & $\approx 12 \mathrm{~kW} / \mathrm{cm}^{2}$ \\
\hline Peak intensity on SESAM & $\approx 16.5 \mathrm{GW} / \mathrm{cm}^{2}$ & $\approx 9.9 \mathrm{GW} / \mathrm{cm}^{2}$ & $\approx 4.8 \mathrm{GW} / \mathrm{cm}^{2}$ \\
\hline Saturation fluence $F_{\text {sat }}$ of SESAM & $112.2 \mu \mathrm{J} / \mathrm{cm}^{2}$ & $61 \mu \mathrm{J} / \mathrm{cm}^{2}$ & $61 \mu \mathrm{J} / \mathrm{cm}^{2}$ \\
\hline Saturation parameter $S$ & $\approx 50-60$ & $\approx 65$ & $\approx 33$ \\
\hline
\end{tabular}

this way, we keep the same intensity on the disk and operate the SESAM at same saturation level. An important tradeoff is a higher demand on the oscillator cavity design given the higher sensitivity toward thermal lensing with larger spot sizes [16]. We can relax these requirements again when we use SESAMs with higher saturation fluences to allow for resonator designs with smaller spot sizes on the SESAM [15].

The excellent power scalability of the thin-disk laser technology should allow kilowatt single-mode oscillators [13] suitable for SESAM modelocking. However, possible SESAM limitations have not been studied so far and contradicting information on damage thresholds and losses has been reported. Previous studies on optimized SESAM designs mostly focused on the realization of low saturation fluences [17], [18], which is important for realizing high repetition rates [19] and stable modelocking of semiconductor lasers [20]. This study resulted in fundamentally modelocked laser oscillators with record-high repetition rates up to $160 \mathrm{GHz}$ in the $1-\mu \mathrm{m}$ spectral region [21] and $100 \mathrm{GHz}$ in the $1.5-\mu \mathrm{m}$ telecom spectral region [22], and the development of new integrated semiconductor lasers [23]. However, SESAMs for ultrafast thin-disk lasers operate in a completely different regime, where pulse energies and average power levels are several orders of magnitude higher. In contrast to low power oscillators, Q-switching instabilities in this case are easily overcome by the very large intracavity pulse energies. In Table I, we present typical SESAM operation parameters in high-power thin-disk oscillators. A useful parameter to describe operation of a SESAM is the saturation parameter $S$

$$
S=\frac{F}{F_{\text {sat }}} .
$$

Typical SESAM modelocked lasers operate at $S=3-10$ [24], but stable operation has been demonstrated in high-energy oscillators at saturation parameters $S>30$ (see Table I). In Table I, we can see that SESAMs in high-power oscillators operate at kilowatt intracavity power levels, peak intensities of tens of gigawatts per square centimeter, and fluences above the millijoule per square centimeter level. Therefore, SESAMs for high-power oscillators require not only large saturation fluences but also high damage thresholds and low nonsaturable losses to avoid thermal effects. Furthermore, the inverse saturable absorption
(ISA) responsible for the reflectivity rollover at high fluences has to be sufficiently low to avoid multiple pulsing [25]-[27]. In this paper, we extend these studies to high-power SESAMs and present the first detailed study on damage thresholds and lifetime of different representative samples. In order to reach the high fluences necessary to carry out this study, we used a high-energy SESAM modelocked Yb:YAG thin-disk oscillator in a high-precision nonlinear reflectivity measurement system to characterize nonlinear reflectivity, induced absorption, and damage of our SESAMs [28], [29]. This allowed us to test our SESAMs up to an unprecedented high fluence level of $0.21 \mathrm{~J} / \mathrm{cm}^{2}$ corresponding to a high peak intensity of $370 \mathrm{GW} / \mathrm{cm}^{2}$.

We designed SESAMs with multiple quantum wells (QWs) in a standard antiresonant configuration and a suitable dielectric topcoating and obtained samples with increased saturation fluence and damage threshold as well as reduced nonsaturable losses and ISA. These samples meet all the requirements for future SESAM modelocked femtosecond oscillators in the kilowatt average output power regime.

\section{ExPerimental Setup ANd MEASUREMENT PROCEDURe}

\section{A. Experimental Setup}

The experimental setup consists of a high-precision nonlinear reflectivity measurement system [29] and a high-power SESAM modelocked $\mathrm{Yb}$ :YAG thin-disk laser oscillator (see Fig. 1). It delivers $15 \mathrm{~W}$ of average power at $10.7-\mathrm{MHz}$ repetition rate corresponding to a pulse energy of $1.4 \mu \mathrm{J}$ in 1 ps pulses.

The variable attenuation stage enables us to access a pulse fluence range of more than four orders of magnitude. We use a highly reflective (HR) mirror with a specified reflectivity of 99.98\% at $1030 \mathrm{~nm}$ (Layertec GmbH, Mellingen, Germany) for reflectivity calibration. A least-squares fit procedure yields the saturation parameters of the SESAM: saturation fluence $F_{\text {sat }}$, modulation depth $\Delta R=R_{\mathrm{ns}}-R_{\text {lin }}$, nonsaturable losses $\Delta R_{\mathrm{ns}}=1-R_{\mathrm{ns}}$, and ISA coefficient $F_{2}$.

Equation (2) describes the SESAM reflectivity for a flattop-shaped beam profile. Our fitting function [28] is based on the following equation but is numerically corrected for a 


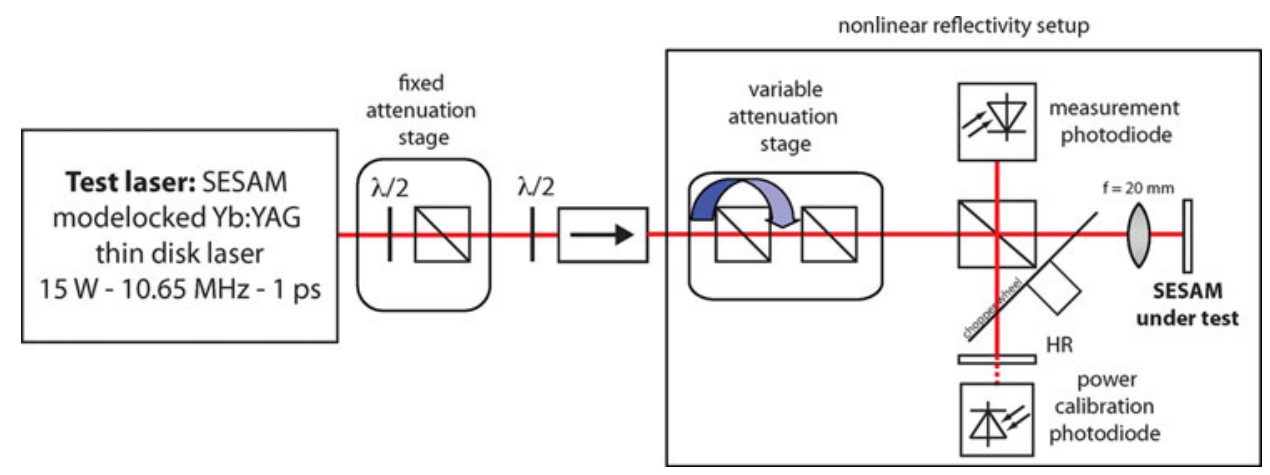

Fig. 1. Experimental setup used for the nonlinear reflectivity measurements and damage characterization of the samples. A high-energy Yb:YAG thin-disk modelocked oscillator is used as the test laser in a high-precision nonlinear reflectivity measurement setup [29]. With a spot diameter on the sample of $17.9 \mu \mathrm{m}$ and with the maximum power available, we were able to achieve pulse fluences up to $0.21 \mathrm{~J} / \mathrm{cm}^{2}$ and peak powers over $370 \mathrm{GW} / \mathrm{cm}^{2}$. HR: highly reflective mirror.

Gaussian-shaped beam:

$$
R(F)=R_{\mathrm{ns}} \frac{\ln \left(1+\left(R_{\mathrm{lin}} / R_{\mathrm{ns}}\right)\left(e^{F / F_{\mathrm{sat}}}-1\right)\right)}{F / F_{\mathrm{sat}}} e^{-F / F_{2}} .
$$

We used a lens with a focal length $f=20 \mathrm{~mm}$ and achieved a spot size diameter of $17.9 \mu \mathrm{m}$ on the sample. At the maximum available power, this resulted in a maximum fluence of $0.21 \mathrm{~J} / \mathrm{cm}^{2}$, which is 30 times higher than previous measurements [29]. The initial fixed attenuation stage was used to set the maximum fluence level for a given measurement in order to benefit from the full 40-dB range of the variable fluence attenuation even for samples with moderate saturation fluences and damage thresholds.

\section{B. Characterization of the ISA}

The choice of appropriate absorber parameters for SESAMs used in high-power thin-disk lasers is crucial to avoid Qswitching instabilities [5] or multipulsing instabilities [25]-[27]. The high intracavity energies involved in passively modelocked thin-disk oscillators combined with soliton modelocking [30], [31] usually make potential Q-switching instabilities not a serious issue. On the other hand, operation at high incident pulse fluences on the SESAM compared to its saturation fluence (i.e., high $S$-parameter levels, see Table I) makes multiple pulsing instabilities a more critical issue. It is, therefore, of particular importance for high-power oscillators to correctly characterize the ISA coefficient $F_{2}$ that describes the additional absorption observed at high fluence levels. We usually refer to this effect as a "rollover" in reflectivity. The $F_{2}$ parameter, typically expressed in $\mathrm{mJ} / \mathrm{cm}^{2}$, describes the curvature of the rollover and is taken into account as an extra parameter in the reflectivity function for the fitting procedure [see (2)].

Another relevant parameter that can be extracted using the nonlinear reflectivity curve is the fluence $F_{0}$, where the maximum reflectivity is reached. These two parameters are related for weak ISA coefficients $\left(F_{0}>5 F_{\text {sat }}\right)$ by [25]

$$
F_{0}=\sqrt{F_{2} F_{\text {sat }} \Delta R} .
$$

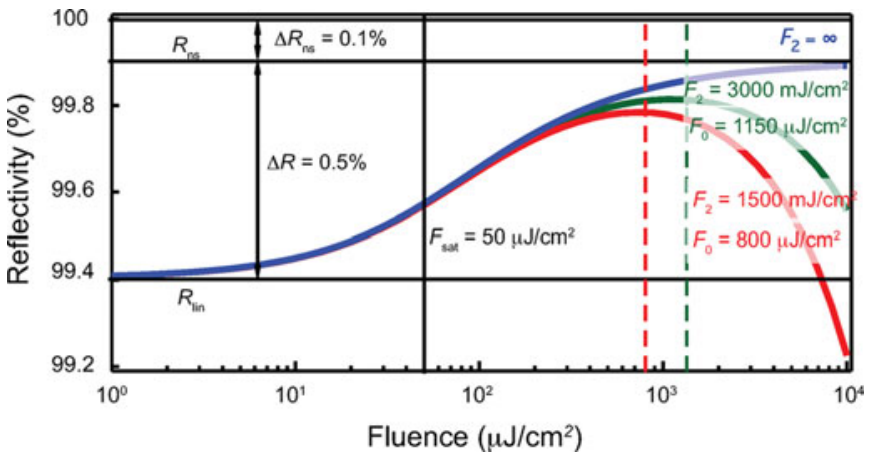

Fig. 2. Influence of different ISA levels on a sample with constant $F_{\text {sat }}=50 \mu \mathrm{J} / \mathrm{cm}^{2}, \Delta R=0.5 \%$, and $\Delta R_{\mathrm{ns}}=0.1 \%$ but with different inverse absorption coefficients $F_{2}$.

Fig. 2 gives an illustration of the influence of the induced absorption coefficient $F_{2}$ when $F_{\text {sat }}, \Delta R$, and $\Delta R_{\text {ns }}$ are kept constant.

The parameter $F_{2}$ can be calculated in the case of two-photon absorption (TPA) and for $\operatorname{sech}^{2}$ pulses using [32], [33]

$$
F_{2}=\frac{\tau_{\mathrm{p}}}{0.585 \int \beta_{\mathrm{TPA}}(z) n^{2}(z)|\varepsilon(z)|^{4} \mathrm{~d} z}
$$

with $z$ being the vertical position within the wafer structure, $\beta_{\text {TPA }}(z)$ is the TPA coefficient expressed in centimeter per gigawatt, $\varepsilon(z)$ is the normalized electric field in the structure, and $n(z)$ is the refractive index. Although $F_{0}$ may appear as a more intuitive parameter, $F_{2}$ is independent of the SESAM parameters, whereas $F_{0}$ depends on the product of $F_{\text {sat }} \cdot \Delta R$. This makes $F_{2}$ a well-suited parameter to compare ISA of different SESAM samples. This formula gives a good approximation for femtosecond pulses. In the case of longer pulses, the observed $F_{2}$ is larger than the calculated one if only TPA is taken into account. The reason for this strong ISA for longer pulses has not yet been clearly elucidated [25].

\section{Design OF THE SESAMs Under TeST}

A straightforward approach to increase the saturation fluence of a SESAM is to grow a highly reflective top mirror on the absorber to increase its finesse [15], [34]. With this approach, the electric field in the absorber layers is reduced, and therefore, 
(a)

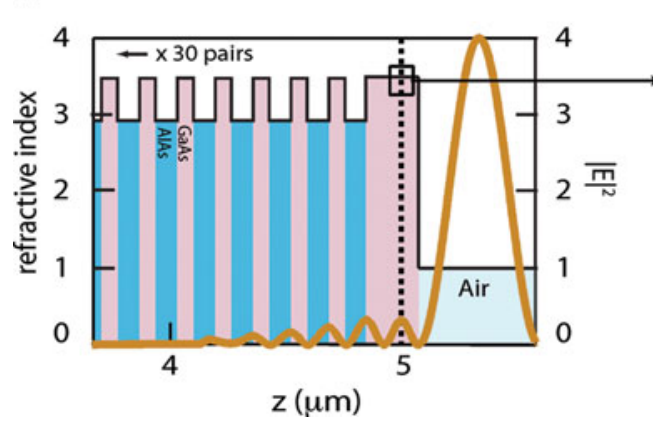

(b)

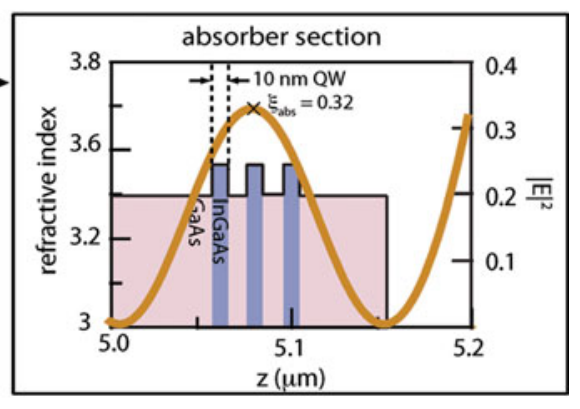

Fig. 3. (a) Non-topcoated SESAM structure and (b) zoom on absorber layers.

(a)

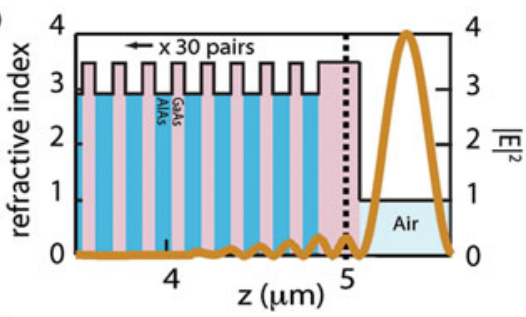

(b)
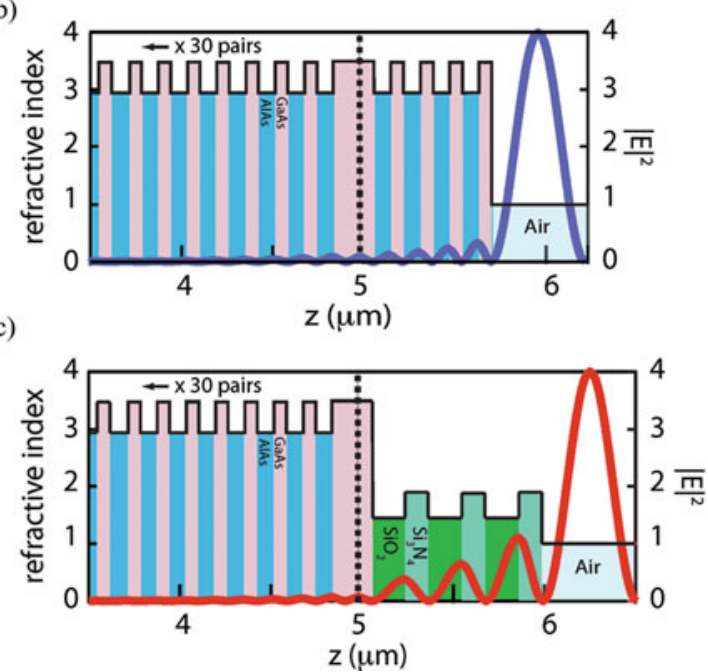

(d)

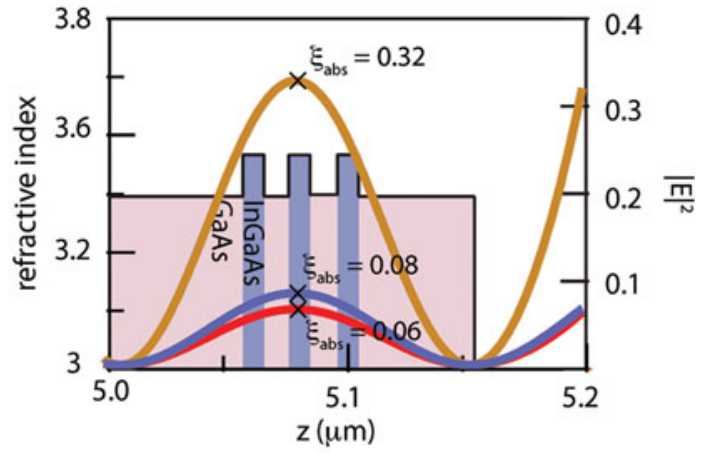

Fig. 4. Overview of the SESAMs under test. (a) SESAM structure without any topcoating (NTC). (b) SESAM structure with a 4-pair quarter-wave layer semiconductor topcoating (SCTC). (c) SESAM structure with a 3-pair quarter-wave layer dielectric topcoating (DTC3). A similar structure consisting of 2-pair quarter-wave layer dielectric topcoating (DTC2) was also used for this study but is not shown in this figure. (d) Field enhancement $\xi$ in the absorber section for the different topcoatings.

the fluence required to saturate the sample is increased. As we increase the saturation fluence, we reduce the modulation depth of the sample since (see Appendix I)

$$
F_{\text {sat }} \Delta R \approx N_{0} h \nu
$$

with $N_{0}$ being the 2-D transparency density of the QW absorber and $h \nu$ is the incident photon energy. We can see in this formula that the product $F_{\text {sat }} \cdot \Delta R$ remains constant for a given absorber since the transparency density is an intrinsic property. Therefore, the samples to topcoat need to have a large enough modulation depth in addition to the basic requirements (i.e., low nonsaturable losses and an initially large saturation fluence). With multiple QWs, we can adjust the mod- ulation depth without changing the saturation fluence of the samples.

In Fig. 3, we show the design of the non-topcoated SESAM (NTC) used for this study. It consists of a 30-pair quarter-wave GaAs/AlAs high finesse distributed Bragg reflector (DBR) and three 10-nm InGaAs QWs as absorber layers. This SESAM was grown by molecular beam epitaxy (MBE) in the FIRST cleanroom facility at ETH Zurich.

The QW section was grown at $T \approx 400{ }^{\circ} \mathrm{C}$. We measured the recovery time of this non-topcoated sample using a standard pump-probe setup. The measurement yielded a recovery time with a slow component of $\tau_{\text {slow }}=202$ ps. The probe pulses used for this measurement had a pulse duration of $\tau_{p}=1 \mathrm{ps}$, which did not allow for the fast component of the SESAM 


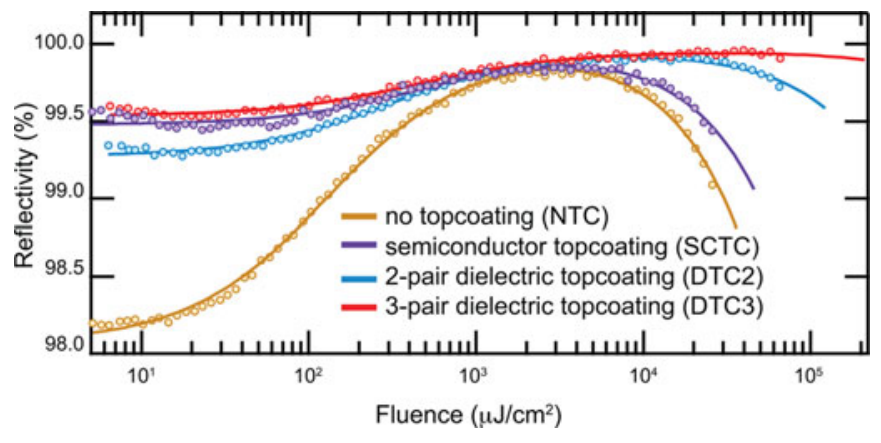

Fig. 5. Nonlinear reflectivity measurements of the SESAMs under test.

response to be resolved. Lower MBE growth temperatures would support shorter recovery times, and therefore, shorter achievable pulse durations [24]. However, the increased number of defects in the growth might lead to samples with higher nonsaturable losses [35]. Since for soliton modelocking, the dependence of the pulse duration with recovery time is proportional to $\tau_{a}{ }^{1 / 4}$ [24], the benefit from a better growth quality with a slightly higher growth temperature is more advantageous than the small benefit from additional pulse shortening with shorter recovery times. Three different topcoatings were grown on these samples (see Fig. 4): in the first case, a semiconductor topcoating was chosen with four pairs of quarter-wave GaAs/AlAs layers grown by MBE [semiconductor topcoating (SCTC), see Fig. 4(b)]. The field enhancement in the absorber is reduced by a factor of $\approx 4$ compared to the uncoated sample and we therefore expect the same increase of the saturation fluence $F_{\text {sat }}$ [see Fig. 4(d)]. For the other cases, a dielectric $\mathrm{SiO}_{2} / \mathrm{Si}_{3} \mathrm{~N}_{4}$ topcoating by plasma-enhanced chemical vapor deposition (PECVD) was chosen. Two sets of samples were coated with two and three pairs of quarter-wave layers [DTC2 and DTC3, see Fig. 4(c)], expecting an increase of the saturation fluence $F_{\text {sat }}$ of 3 and 5, respectively [see Fig. 4(d)]. These topcoatings are particularly attractive because the deposition process is much easier and considerably more cost efficient than an MBE topcoating. From a material point of view, the TPA coefficients of $\mathrm{SiO}_{2}$ and $\mathrm{Si}_{3} \mathrm{~N}_{4}$ are also much lower than those of GaAs and AlAs because of the much higher bandgap energy of dielectrics, which makes them better suited for reduced ISA [36].

\section{Nonlinear Reflectivity Measurement Results}

The nonlinear reflectivity response and different measured parameters of the SESAMs under test are presented in Fig. 5 and Table III.

Fig. 5 shows the effect of the different topcoatings: as the saturation fluence increases, the modulation depth decreases by a similar factor. The saturation fluences of all the topcoated samples are $>150 \mu \mathrm{J} / \mathrm{cm}^{2}$ and their modulation depths are between 0.4 and $0.8 \%$. SESAMs used in high-power thin-disk lasers typically have modulation depths in this range [6], [15], [37]. This makes all our fabricated SESAMs excellent candidates for high-power oscillator modelocking. The 3-QW non-topcoated sample with $2 \%$ modulation depth is ideally suited for topcoatings designed for a saturation fluence increase of 3-5. All
TABLE II

MeAsured $F_{2}$ AND CALCUlated $F_{2}$ TAKing into Account OnLY TPA

\begin{tabular}{lccc}
\hline \hline SESAM & $\begin{array}{c}F_{2, \text { meas }} \\
\left(\mathrm{mJ} / \mathrm{cm}^{2}\right)\end{array}$ & $\begin{array}{c}F_{2, \text { TPA }} \\
\left(\mathrm{mJ}^{2} \mathrm{~cm}^{2}\right)\end{array}$ & $F_{2, \text { TPA }} / F_{2, \text { meas }}$ \\
\hline NTC & 3200 & 8200 & 2.56 \\
SCTC & 5523 & 15000 & 2.71 \\
DTC2 & 31700 & 70200 & 2.21 \\
DTC3 & 346000 & 206000 & 0.6 \\
\hline \hline
\end{tabular}

NTC: No topcoating, SCTC: semiconductor topcoating with four quarter-wave pairs of GaAs/AlAs, and DTC2 and DTC3: dielectric top coating with two and three quarter-wave pairs of $\mathrm{SiO}_{2} / \mathrm{Si}_{3} \mathrm{~N}_{4}$, respectively

SESAMs have negligible nonsaturable losses $<0.1 \%$ and therefore reduced thermal load.

All topcoated SESAMs have increased ISA coefficients compared to that of the non-topcoated sample. In the case of the semiconductor topcoated SESAM, a small increase is observed. This is due to the fact that GaAs has a strong TPA coefficient and even if the field in the DBR structure and the absorber section is reduced, the GaAs/AlAs topsection experiences a strong electric field. In the case of the dielectric topcoatings, the induced absorption coefficient $F_{2}$ is significantly increased. For the 2-layer and 3-layer dielectric topcoatings, we measured an increase of the ISA coefficient of one and two orders of magnitude, respectively. This is due to the significantly lower TPA coefficient of the dielectric material compared to the high TPA coefficient of GaAs. Therefore, SESAMs with similar saturation parameters (similar electric field distributions in the DBR and the absorber region) but different topcoatings have different ISA responses. Using (4), we calculated the expected $F_{2}$ parameter taking into account only TPA for all our fabricated structures and assuming a pulse duration of $\tau_{p}=1 \mathrm{ps}$. For this calculation, the TPA coefficients of $\mathrm{AlAs}, \mathrm{SiO}_{2}$, and $\mathrm{Si}_{3} \mathrm{~N}_{4}$ are considered negligible compared to that of $\mathrm{GaAs}$, and we used $\beta_{\mathrm{TPA}, \mathrm{GaAs}}=$ $20 \mathrm{~cm} / \mathrm{GW}$ [36]. The results are summarized in Table II.

For the SESAM with the 3-pair dielectric topcoating (DTC3), the available power limits the measurement accuracy of $F_{2}$. As we can see in Fig. 5, the maximum fluence does not allow us to measure deep enough into the rollover regime; therefore, $F_{2}$ is probably overestimated for this SESAM. For all other SESAMs, there is a factor of $\approx 2.5$ between the calculated and measured $F_{2}$ indicating that the rollover observed is stronger than that predicted by TPA for this pulse duration. This is due to the relatively long pulses we used in the study. Indeed, in [25] it was shown that the rollover observed in the picosecond regime was stronger than that predicted by TPA only in similar structures. The observed factor of around 2-3 seems to be in good agreement with this previous study carried out with 3-ps long pulses (see Table II).

\section{DAMAGE AND LifETIME INVESTIGATION}

\section{A. Damage Fluence and Lifetime Measurements}

We define damage of a SESAM under test as an irreversible change in the structure resulting in a dramatic drop in the measured reflectivity. The damage fluence threshold is then defined 
TABLE III

EXTRACTED PARAMETERS From tHe NONLINEAR REFLECTIVITY MEASUREMENT FOR THE DifFERENT SESAMs UNDER TEST

\begin{tabular}{ccccccccc}
\hline \hline SESAM & $\begin{array}{c}F_{\text {sat }} \\
\left(\mu \mathrm{J} / \mathrm{cm}^{2}\right)\end{array}$ & $\begin{array}{c}\Delta R \\
(\%)\end{array}$ & $\begin{array}{c}\Delta R_{\text {ns }} \\
(\%)\end{array}$ & $\begin{array}{c}F_{2} \\
\left(\mathrm{~mJ} / \mathrm{cm}^{2}\right)\end{array}$ & $\begin{array}{c}F_{0} \\
\left(\mathrm{~mJ} / \mathrm{cm}^{2}\right)\end{array}$ & $\begin{array}{c}F_{\text {sat }} \cdot \Delta R \\
\left(\mu \mathrm{J} / \mathrm{cm}^{2}\right)\end{array}$ & $\begin{array}{c}\text { Expected } \\
\text { increase }\end{array}$ & $\begin{array}{c}\text { Obtained } \\
\text { increase }\end{array}$ \\
\hline NTC & 72 & 2.05 & 0.04 & 3200 & 3.1 & 1.44 & - & - \\
SCTC & 279 & 0.52 & 0.01 & 5523 & 3.3 & 1.45 & $\approx 4$ & 3.9 \\
DTC2 & 168 & 0.71 & 0.02 & 31700 & 8.7 & 1.19 & $\approx 3$ & 2.3 \\
DTC3 & 247 & 0.43 & 0.04 & 346000 & 30.2 & 1.06 & $\approx 5$ \\
\hline \hline
\end{tabular}

NTC: No topcoating, SCTC: semiconductor topcoating with four quarter-wave pairs of GaAs/A1As, and DTC2 and DTC3: dielectric topcoating with, respectively, two and three quarter-wave pairs of $\mathrm{SiO}_{2} / \mathrm{Si}_{3} \mathrm{~N}_{4}$

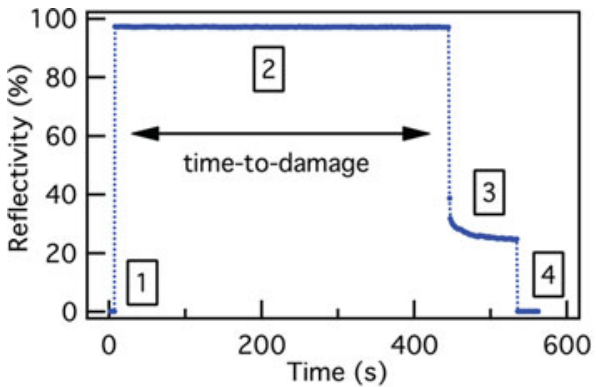

(a)

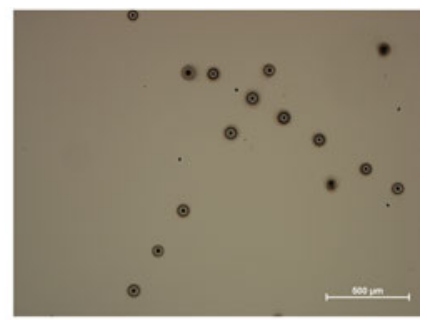

(b)

Fig. 6. (a) Typical time-to-damage measurement. During (1), the sample is blocked, and at (2) the sample is exposed to the laser beam. Once damage has occurred, the reflectivity drops [in this case to $\approx 30 \%$ (3)]. The laser beam on the SESAM under test is blocked (4) and the SESAM is moved laterally to perform the next measurement. In this example, we measured a time-to-damage of $445 \mathrm{~s}$ at a fluence of $27 \mathrm{~mJ} / \mathrm{cm}^{2}$, corresponding to a fluence $65 \%$ lower than the damage threshold. (b) Image of a tested SESAM where we can see multiple damage spots resulting from this damage characterization.

as the minimum fluence where this irreversible reflectivity drop occurs in $<1 \mathrm{~s}$.

In order to measure the damage fluence and the time-todamage of a sample, we use the setup described in Section IIA. We set the fluence to a constant value and track reflectivity of the sample versus time. A typical measurement trace is shown in Fig. 6(a). First, the fluence is set to the desired value while the sample arm is blocked (1). The measurement of reflectivity versus time is then started, and the laser is focused onto the sample. The time before the irreversible reflectivity drop (2) is what we define as "time-to-damage". One reflectivity measurement point lasts typically around $1 \mathrm{~s}$, and the precision of the measurement is, therefore, limited to this duration. The observed drop in reflectivity is stronger when damage occurs at higher fluences, but typically the drop is of $50-90 \%$ of the initial reflectivity of the nondamaged sample.

In order to find the damage threshold, we first scan the fluence in steps of approximately $5 \mathrm{~mJ} / \mathrm{cm}^{2}$ starting at around $100 \times F_{\text {sat }}$ based on typical damage values. Once a fluence is found where instantaneous damage is observed, we perform a fine scan both in fluence (steps of $\approx 1 \mathrm{~mJ} / \mathrm{cm}^{2}$ ) and in the $z$ position of the sample in order to find the exact focus position for minimum damage fluence. The obtained minimum value is what we define as damage threshold fluence of a sample.

Once a spot has been damaged, we move the SESAM under test in the $x$ - or $y$-direction in order to perform the next measurement on an undamaged spot. It is interesting to note that the higher the damage threshold, the bigger the impact trace on the SESAM when damaged; therefore, a larger translation to a new spot is required to perform the next measurement. A large translation of the SESAM under test might result in slight differences in the SESAM parameters due to a small linear layer thickness variation of less than $<1 \%$ along the full wafer resulting from the MBE growth. We limited this effect with a maximum translation of $500 \mu \mathrm{m}$ on the sample.

\section{B. Damage of Different Topcoated SESAMs}

As a first step, we performed damage tests on the different 3-QW SESAMs described in Section III to compare the effect of the different topcoatings on the damage behavior. The damage fluences of these samples are presented in Table IV together with the different parameters of the tested SESAMs.

The topcoated SESAMs show, in all cases, higher damage fluences than without a topcoating. The damage fluences for the dielectric topcoatings are much higher than for the semiconductor topcoated SESAM with similar parameters. It is interesting to note that regardless of the sample, instantaneous damage occurs at fluences deep in the rollover regime, where SESAM modelocked lasers would not operate in a stable regime (e.g., saturation parameters $S>150$ ). For our sample with three quarter-wave pairs of $\mathrm{SiO}_{2} / \mathrm{Si}_{3} \mathrm{~N}_{4}$ topcoating, we could not observe damage even at the available maximum fluence of $0.21 \mathrm{~J} / \mathrm{cm}^{2}$ in our setup. This corresponds to a peak intensity of $370 \mathrm{GW} / \mathrm{cm}^{2}$ on the sample. This particular sample was tested at this maximum fluence level for several hours and no damage was observed. We measured lifetime curves for the samples where the damage fluence could be measured and the results are presented in Fig. 7.

For the different samples, the lifetime curves seem to follow the same exponential behavior suggesting a similar damage 
TABLE IV

MEASured Damage Parameters

\begin{tabular}{|c|c|c|c|c|c|c|c|}
\hline SESAM & $\begin{array}{c}F_{\text {sat }} \\
\left(\mu \mathrm{J} / \mathrm{cm}^{2}\right)\end{array}$ & $\begin{array}{l}\Delta R \\
(\%)\end{array}$ & $\begin{array}{l}\Delta R_{\text {ns }} \\
(\%)\end{array}$ & $\begin{array}{c}F_{2} \\
\left(\mathrm{~mJ} / \mathrm{cm}^{2}\right)\end{array}$ & $\begin{array}{c}F_{0} \\
\left(\mathrm{~mJ} / \mathrm{cm}^{2}\right)\end{array}$ & $\begin{array}{c}F_{\mathrm{d}} \\
\left(\mathrm{mJ} / \mathrm{cm}^{2}\right)\end{array}$ & $S=F_{\mathrm{d}} / F_{\text {sat }}$ \\
\hline NTC & 72 & 2.05 & 0.04 & 3200 & 3.1 & 32.6 & 452 \\
\hline SCTC & 279 & 0.52 & 0.01 & 5523 & 3.3 & 44.1 & 157 \\
\hline DTC2 & 168 & 0.71 & 0.02 & 31700 & 8.7 & 122 & 726 \\
\hline DTC3 & 247 & 0.43 & 0.04 & 346000 & 30.2 & $>210$ & $>850$ \\
\hline
\end{tabular}

NTC: No topcoating, SCTC: semiconductor topcoating with four quarter-wave pairs of GaAs/AlAs, and DTC: dielectric topcoating with two and three quarter-wave pairs of $\mathrm{SiO}_{2} / \mathrm{Si}_{3} \mathrm{~N}_{4}$.

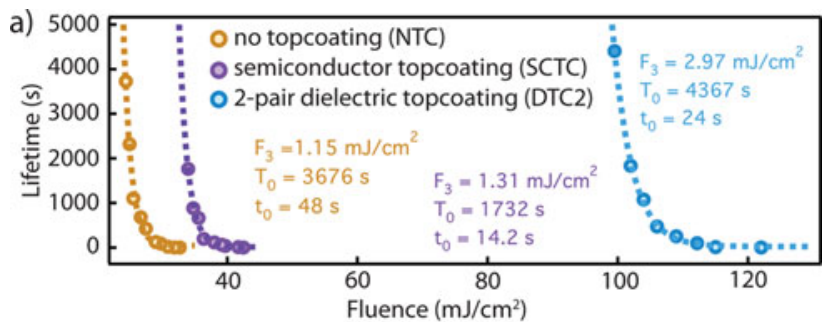

(a)

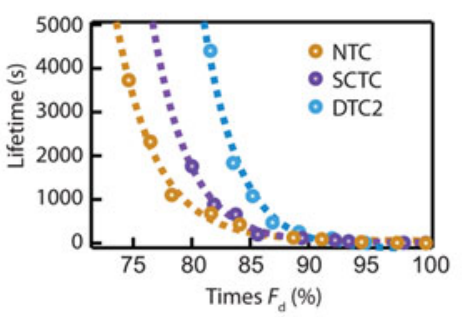

(b)

Fig. 7. Lifetime curves of different SESAMs: (a) lifetime curves with fluence axis in absolute value for the non-topcoated SESAM with three QWs (NTC, brown), the same SESAM with a GaAs/AlAs semiconductor topcoating (SCTC, purple), and the same SESAM with a $\mathrm{SiO}_{2} / \mathrm{Si}_{3} \mathrm{~N}_{4}$ dielectric topcoating (DTC2, blue). (b) Lifetime curves in reference to the instantaneous damage fluence of the respective SESAMs.

TABLE V

Calculated Damage Parameters Using THE FIT FUNCTION DESCRIBED IN (6)

\begin{tabular}{cccccc}
\hline \hline SESAM & $\begin{array}{c}F_{\text {sat }} \\
\left(\mu \mathrm{J} / \mathrm{cm}^{2}\right)\end{array}$ & $\begin{array}{c}F_{3} \\
\left(\mathrm{~mJ} / \mathrm{cm}^{2}\right)\end{array}$ & $\begin{array}{c}F_{0} \\
\left(\mathrm{~mJ} / \mathrm{cm}^{2}\right)\end{array}$ & $t\left(F_{\mathrm{d}} / 2\right)$ & $t\left(F_{0}\right)$ \\
\hline NTC & 72 & 1.15 & 3.1 & $\begin{array}{c}4.210^{6} \mathrm{~s} \\
(48 \text { days })\end{array}$ & $4.010^{11} \mathrm{~s}$ \\
SCTC & 279 & 1.31 & 3.3 & $\begin{array}{c}1.610^{7} \mathrm{~s} \\
(185 \text { days })\end{array}$ & $2.510^{13} \mathrm{~s}$ \\
DTC2 & 168 & 2.97 & 8.7 & $\begin{array}{c}1.810^{9} \mathrm{~s} \\
(57 \text { years })\end{array}$ & $8.110^{16} \mathrm{~s}$ \\
DTC3 & 247 & $*$ & 30.2 & $*$ & $*$ \\
\hline \hline
\end{tabular}

*No damage threshold could be measured for the three-pairs DTC SESAM. NTC: Nontopcoating, SCTC: semiconductor topcoating with a four quarter-wave pairs of GaAs/AlAs, DTC2 and DTC3: dielectric topcoating with two or three quarter-wave pairs of $\mathrm{SiO}_{2} / \mathrm{Si}_{3} \mathrm{~N}_{4}$, respectively.

mechanism in this fluence range. The fit for the lifetime curves was done using a single exponential function

$$
t(F)=t_{0}+T_{0} \mathrm{e}^{-\frac{\left(F-F_{\text {offset }}\right)}{F_{3}}} .
$$

The fit parameters are presented with the lifetime curves in Fig. 7. For every SESAM, the measurements were done for fluence levels ranging from the instantaneous damage fluence

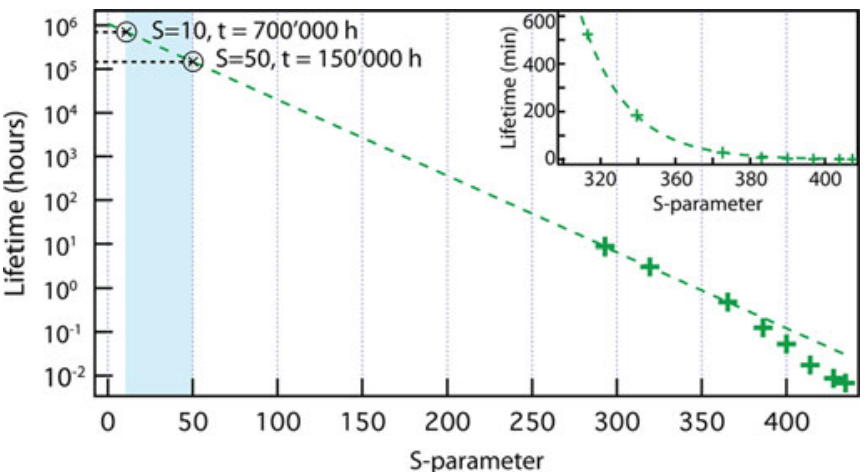

Fig. 8. SESAM with a single QW absorber. Lifetime of the sample is presented in a logarithmic scale and as a function of its saturation parameter. Zone marked in blue is the regime of typical operation in high-power oscillators. At these fluences, the suggested lifetimes are in the order of several hundred thousand hours.

to around $80 \%$ of this value, leading to time-to-damage values of 1-2 h. The parameter $F_{3}$ is a representative value to compare the different samples. It represents the fluence at which the lifetime has dropped to $1 / e$ of the zero fluence lifetime value.

We can use these lifetime curves to evaluate the lifetime of a SESAM at a given lower fluence. The maximum fluence at which a SESAM can be used for modelocking is the point $F_{0}$ where the maximum reflectivity is reached. It is, therefore, interesting to evaluate the lifetime at this fluence. We also present the values of these lifetimes to half the damage fluence. The different values for the measured SESAMs are presented in Table V.

The extended lifetime curves suggest lifetimes of several years at the maximum reflectivity fluence $F_{0}$. At more typical operation points, the lifetimes would be even larger. Clearly, other damaging mechanisms need to be considered at lower fluences, and this single exponential most likely will not be enough to fully characterize the long-term lifetime of a SESAM. Therefore, measurements at much lower fluence levels are necessary in a similar study.

\section{Extension of the Lifetime Curves to Lower Fluences}

We measured lifetime values of $>8.7 \mathrm{~h}$ for one sample with one QW as an absorber. This lifetime is presented in Fig. 8 as a function of the saturation parameter and extended to very low saturation parameters. We can see that at saturation parameters of $10<S<50$, the suggested lifetimes are in the order of $700000-150000 \mathrm{~h}$ which is approximately an order of 


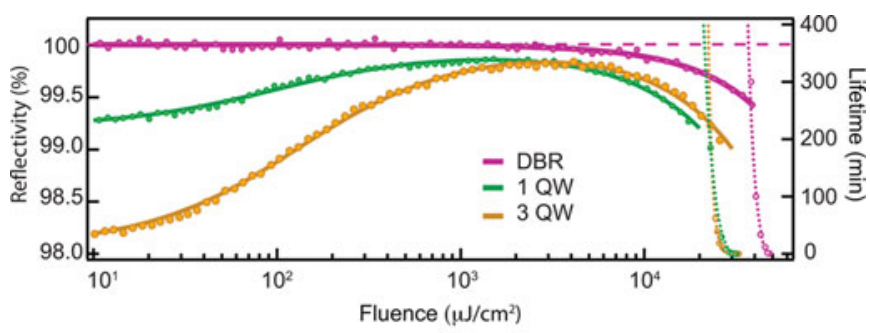

(a)

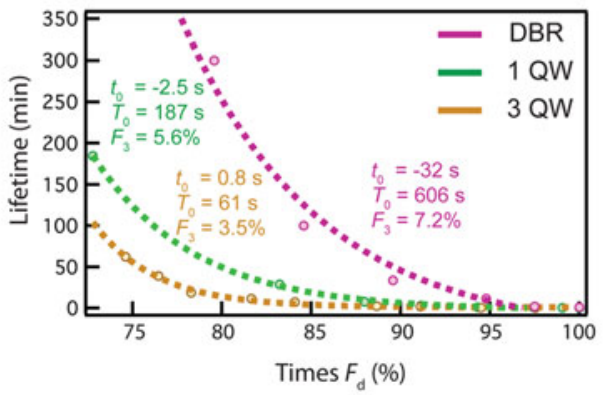

(b)

Fig. 9. (a) Lifetime curves and nonlinear reflectivity of a DBR (pink), a nontopcoated single-QW SESAM (green), and a non-topcoated 3-QW SESAM (brown). (b) Lifetime curves in reference to the instantaneous damage fluence of the respective SESAMs.

magnitude larger than observed in some high-power commercial systems using SESAM technology [38].

This seems to indicate that another mechanism has to be taken into account for much lower fluences. Nevertheless, considering that the longest time-to-damage point was taken at a fluence over an order of magnitude larger than the evaluated points, the model seems to give a good approximation of the lifetime of SESAMs. However, we can see here that it is crucial to take points at long lifetimes $(t>10 \mathrm{~h})$ for a correct extension of the lifetime curves.

\section{Influence of the Absorber Section on the Lifetime: DBR, $1 Q W$, and $3 Q W$}

In order to identify the damage mechanism, we compared the damage behavior of a SESAM with a single QW absorber, a 3-layer QW absorber (same one described in Section III), and the DBR mirror (without absorber section) in order to evaluate if the damage thresholds are dependent on the saturable absorber properties. All the samples have no topcoating. The results are presented in Fig. 9 and Table VI.

We can see that the damage behavior for a DBR is similar to that of the characterized SESAMs. Damage occurs deep in the rollover regime, and this rollover occurs at comparable fluence levels as for the SESAMs. This confirms that this mechanism is related to the ISA process, which is mainly caused by the GaAs layers in the structure. We can see in Fig. 9(b) that the absorbers seem to have a tendency to slightly reduce the lifetime. This could be due to the fact that the SESAMs have additional GaAs spacers in the absorber section where the electric field is very strong, and this represents an important contribution to the ISA. This would also explain the $50 \%$ higher damage fluence for the DBR (Table VI). Using AlAs barrier layers could, therefore, increase the damage threshold of these non-topcoated SESAMs.
TABLE VI

DAMAGE PARAMETERS MEASURED FOR A DBR (PINK) A NON-TOPCOATED SINGLE-QW SESAM (GREEN) AND A NON-TOPCOATED 3-QW SESAM (BROWN)

\begin{tabular}{ccccc}
\hline \hline Sample & $\begin{array}{c}F_{\text {sat }} \\
\left(\mu \mathrm{J} / \mathrm{cm}^{2}\right)\end{array}$ & $\begin{array}{c}F_{2} \\
\left(\mathrm{~mJ} / \mathrm{cm}^{2}\right)\end{array}$ & $\begin{array}{c}F_{\mathrm{d}} \\
\left(\mathrm{mJ} / \mathrm{cm}^{2}\right)\end{array}$ & $\begin{array}{c}F_{3} \\
\left(\% \text { of } F_{\mathrm{d}}\right)\end{array}$ \\
\hline DBR & 0 & 7400 & 48 & 7.2 \\
1-QW & 57 & 2600 & 32.1 & 5.6 \\
3-QW & 72 & 3200 & 32.6 & 3.5 \\
\hline \hline
\end{tabular}

\section{E. Damage Mechanism}

In Fig. 10, we show the damage curves of the SESAMs described in Section III (see Fig. 4) simultaneously with the reflectivity curves. For all SESAMs under test, damage occurs at fluences deep in the rollover regime, suggesting a damage mechanism related to the absorbed energy due to the ISA rollover. It is interesting to note that in the case of DTC2 where $F_{2}$ is greatly increased, the damage curve is also shifted to higher values. The energy per area absorbed by the sample due to the ISA process is

$$
F_{\mathrm{abs}} \approx \frac{F^{2}}{F_{2}} .
$$

This formula is valid for $F>>F_{\text {sat }}, F<<F_{2}$ and small nonsaturable losses (see Appendix II). Therefore, if we consider that damage occurs at a given level of deposited energy, the damage fluence $F_{d}$ for all SESAMs scales proportionally to $\sqrt{F_{2}}$. In the case of SESAMs with the same absorber, where the product $F_{\text {sat }} \cdot \Delta R$ is constant, the damage fluence $F_{d}$ scales proportionally to $F_{0}$ as shown in Appendix II.

In order to illustrate this dependence, we plotted the ratio $F_{d} / F_{0}$ for four different SESAM topcoating designs (see Fig. 11). All SESAMs are based on the 3-QW absorber described in the previous section and therefore have the same absorber structure with a constant $F_{\text {sat }} \cdot \Delta R$ product. However, even for the same topcoating design, we observe differences in the ISA response of the samples due to the inhomogeneity of the MBE growth with respect to the center position of the wafer. In total, we measured 13 samples at several positions.

These results clearly indicate that the ratio $F_{d} / F_{0}$ remains constant. This means that even for different topcoatings, the damage threshold seems to scale proportionally to $F_{0}$ in the case of SESAMs with the same absorber section where the product $F_{\text {sat }} \cdot \Delta R$ is constant. If we want to extend this evaluation to samples with different absorbers, and even to a DBR mirror (since the absorber does not seem to be responsible for the damage mechanism), it is relevant to compare the damage threshold not to $F_{0}$ but to $\sqrt{F_{2}}$ and to verify $F_{d} \propto \sqrt{F_{2}}$. In Fig. 12, we plotted the ratio $\sqrt{F_{2}} / F_{d}$ for 16 different samples for which we could measure the damage fluence.

The data seem to confirm that the damage process is dominated by the energy absorbed by the sample due to the ISA mechanism, and that the main contribution to this ISA comes from the field in the DBR and spacer layers and not by the absorber. This gives a clear indication on the damage behavior of such SESAMs, suggesting that catastrophic damage occurs due 


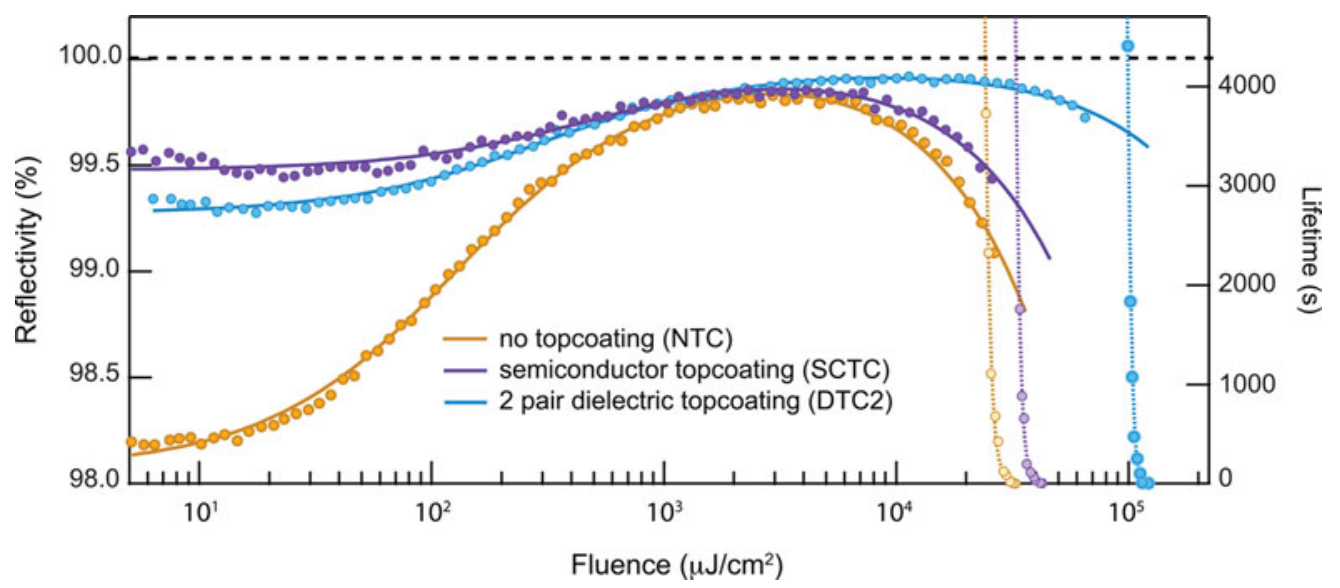

Fig. 10. Lifetime curves plotted with the nonlinear reflectivity curves of the corresponding SESAMs.

to heating of the lattice by energy absorbed by the ISA process. It also indicates a guideline on how to shift the damage fluence to higher values by simply increasing $F_{2}$.

\section{High DAMAGE-Threshold SESAM DESIGN GUIDELINES}

We have identified two important parameters concerning catastrophic damage of SESAMs at high fluences. First, the damage mechanism originates in the amount of energy deposited by ISA in the sample. The damage threshold scales, therefore, with $\sqrt{F_{2}}$. In our study, we used 1-ps pulses; therefore, the mechanism involved is dominated by TPA. Another mechanism that could be partly responsible for damage at these high fluences is, for example, hot-carrier generation. We also demonstrated that the absorber itself is not responsible for the damage mechanism, since a DBR mirror showed comparable damage threshold and lifetime behavior to those of the different SESAMs. We can, therefore, give guidelines to develop SESAMs for operation in high-power oscillators.

1) Multiple QWs allow for tuning of the modulation depth without changing the saturation fluence. This is required in order to have an initially large $\Delta R$ without topcoating to compensate for the lower $\Delta R$ with topcoating. In the case of a relatively low number of QW, where the absorbers can be placed simultaneously in one antinode of the electric field, this has a small influence on the damage threshold because of the additional GaAs spacer layers used as barriers for the InGaAs QW absorbers. In the case of a large number of QW, the amount of material to add becomes significant. In this case, one could also consider using another material with lower $\beta_{\mathrm{TPA}}$ (for instance AlAs) for such QW barrier layers. Without GaAs barrier layers, we would expect a damage behavior of the non-topcoated SESAMs almost identical to that of a DBR mirror.

2) The absorber section of the samples used for this study was grown at $T \approx 400{ }^{\circ} \mathrm{C}$ leading to recovery times in the order of $\approx 200 \mathrm{ps}$. In thin-disk lasers, low-temperature grown SESAM recovery times are typically in the order of $\tau \approx 10-300$ ps. Lower growth temperatures would not only result in shorter recovery times but also in a larger growth defect density [35]. The chosen growth temperature

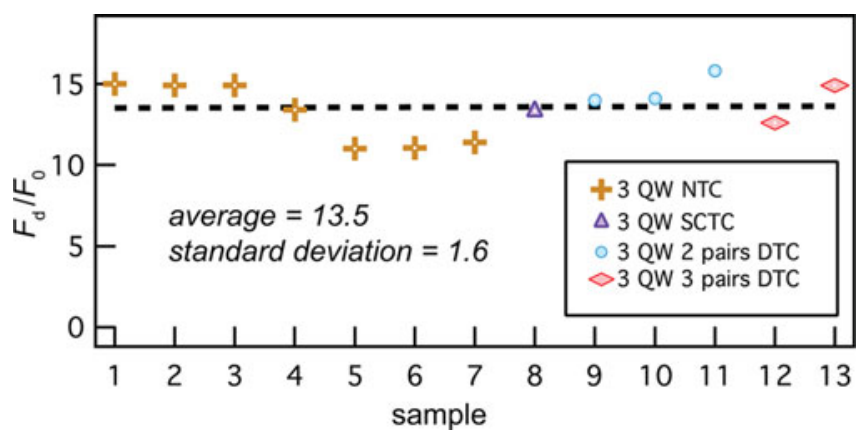

Fig. 11. Ratio $F_{d} / F_{0}$ for 13 different 3-QW SESAMs with different topcoatings.

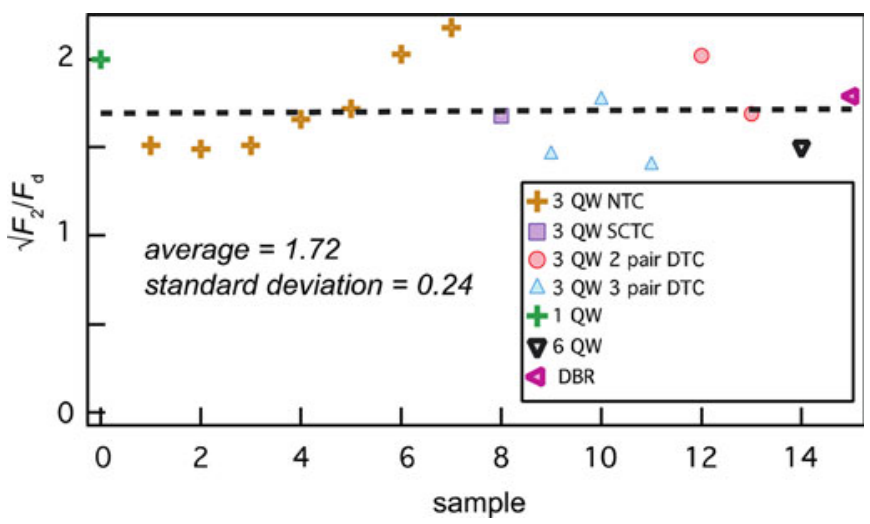

Fig. 12. Ratio $\sqrt{F_{2}} / F_{\text {d }}$ for 16 different samples. We considered here samples with 1,3, and 6 QWs and a DBR mirror. Samples with three QWs with different topcoatings are also compared and plotted.

seems to represent a good compromise between recovery time and growth defects that could further reduce the damage thresholds. However, the exact influence of this growth temperature on the damage behavior still needs to be studied. In this case, in spite of the relatively low growth temperature, we have demonstrated that the damage threshold and parasitic losses of the DBR are similar to those of the 3-QW and 1-QW SESAMs, showing a sufficiently good growth quality.

3) Dielectric topcoatings are preferred over semiconductor topcoatings to increase the saturation fluence. The 
topsection of a SESAM is critical because it experiences a very strong electric field and therefore contributes strongly to the ISA and to the damage mechanism. By using a dielectric material, we achieve larger values for $F_{2}$ and, consequently, higher damage fluences.

\section{CONCLUSION AND OUTLOOK}

We have presented in this paper, to the best of our knowledge, the first detailed study on damage of SESAMs designed for high intracavity energy oscillators. We have identified the relevant nonlinear parameters to compare SESAM damage independently of their specific design structure. We have clearly demonstrated a dependence of the damage threshold on the ISA parameter $F_{2}$ and the maximum reflectivity fluence $F_{0}$, by demonstrating that the damage fluence $F_{\mathrm{d}}$ for all SESAMs scales with $\sqrt{F_{2}}$. Additionally, we have shown that the main contribution to this damage process comes from the GaAs layers in the SESAM structure and not from the absorber layers. In the case of SESAMs with the same absorber (i.e., constant $\left.F_{\text {sat }} \cdot \Delta R\right)$, the damage fluence $F_{\mathrm{d}}$ scales proportionally to $F_{0}$. Therefore, damage occurs due to heating of the lattice by energy absorbed because of ISA and not by the QW absorbers. Additional damage measurements on a simple semiconductor DBR mirror further confirm this conclusion. We give clear design guidelines for SESAMs with reduced ISA and, therefore, reduced damage thresholds.

We could demonstrate SESAMs with high saturation fluences of $>200 \mu \mathrm{J} / \mathrm{cm}^{2}$, low nonsaturable losses of $<0.1 \%$, reduced ISA, and reduced damage threshold. We achieved this by using a dielectric topcoating on a multiple-QW SESAM. For the SESAM with a saturation fluence of $247 \mu \mathrm{J} / \mathrm{cm}^{2}$, nonsaturable losses of $\Delta R_{\mathrm{ns}}=0.01 \%$, and a modulation depth of $\Delta R=0.43 \%$, we could not observe damage even at fluences as high as $0.21 \mathrm{~J} / \mathrm{cm}^{2}$ — the maximum available fluence in our setup. This corresponds to peak intensities of $370 \mathrm{GW} / \mathrm{cm}^{2}$. For all SESAMs, the damage threshold occurs at fluences where it is not possible to operate a modelocked laser in stable operation.

Time-to-damage measurements were done and lifetime curves of $>8 \mathrm{~h}$ were measured for different SESAMs, confirming a clear exponential behavior and suggesting lifetimes of several years at standard operation fluences. However, at much lower fluences than the instantaneous damage fluence, other damage effects might have to be considered. Nevertheless, these extrapolated lifetimes are only one order of magnitude higher than those observed in some high-power commercial systems using SESAM technology [38].

Future damage investigations will explore different laser operation regimes such as pulse duration, pulse repetition rate, and MBE growth temperature of the absorber. Another interesting investigation would consist of performing a similar study to determine the damage mechanisms involved in carbon nanotube absorbers [39]-[42], and compare damage behavior of both absorbers.

It is of particular interest to reproduce a similar study at shorter pulse duration such as 100 fs. However, with the current guidelines, it is already possible to estimate the damage threshold at shorter pulse durations, even though these values still need to be experimentally confirmed.

$$
\begin{gathered}
\text { APPENDIX I } \\
F_{\text {sat }} \cdot \Delta R=\text { CONSTANT [43] }
\end{gathered}
$$

We will demonstrate here that the product of modulation depth times the saturation fluence is proportional to the transparency density $N_{0}$. The transparency density $N_{0}$ depends on the material composition, wavelength, temperature, and confinement.

The condition for transparency is to have equal probability for stimulated emission and absorption. In terms of intrinsic parameters, one can show that this condition is fulfilled when the separation between the quasi-Fermi levels equals the photon energy, i.e.,

$$
\mathrm{E}_{f, c}-\mathrm{E}_{f, v}=h \nu
$$

The number of electrons should equal the number of holes, i.e., $n=p$. The carrier densities are computed with density of states $D(E)$ and the Fermi functions $f_{c}$ and $f_{v}$ for conduction and valence band, respectively,

$$
\begin{aligned}
& n=\int_{E_{\mathrm{c}}}^{\infty} D(E) f_{\mathrm{c}}(E) \mathrm{d} E \\
& p=\int_{-\infty}^{E_{\mathrm{v}}}\left[D(E)\left(1-f_{\mathrm{v}}(E)\right)\right] \mathrm{d} E \\
& n=p=N_{0}
\end{aligned}
$$

with the Fermi functions $f_{c}$ and $f_{v}$ depending on the Fermi energies $E_{f, c}$ and $E_{f, v}$, respectively. The transparency density can, therefore, be calculated by computing the Fermi levels numerically.

The fluence needed to obtain transparency can be obtained by multiplying the transparency density by the photon energy and the thickness of the considered layer in the case of bulk semiconductor

$$
F_{\mathrm{t}}=d h \nu N_{0} .
$$

In the case of QWs, we just need to replace the transparency density by the 2-D transparency density $N_{0,2 \mathrm{D}}$, which is calculated with the modified density of states for QW $D_{\mathrm{QW}}(E)$

$$
F_{\mathrm{t}}=d h \nu N_{0,2 \mathrm{D}} F_{\mathrm{abs}}=F(1-R(F)) .
$$

We can also express the pulse fluence needed for transparency in terms of extrinsic SESAM parameters. The absorbed pulse energy per area for a pulse with fluence $F$

$$
\begin{aligned}
F_{\mathrm{abs}} & =F(1-R(F)) \\
R(F) & =R_{\mathrm{ns}} \frac{\ln \left(1+\frac{R_{\mathrm{lin}}}{R_{\mathrm{ns}}}\left(e^{\frac{F}{F_{\mathrm{sat}}}}-1\right)\right)}{\frac{F}{F_{\mathrm{sat}}}} e^{-\frac{F}{F_{2}}} .
\end{aligned}
$$

For a saturated SESAM $(F \rightarrow \infty)$ and using $(\mathrm{A} 7)$ and (A8)

$$
\begin{aligned}
& F_{\mathrm{t}}=\lim _{F \rightarrow \infty} F(1-R(F)) \\
& F_{\mathrm{t}}=\ln \left(\frac{1}{R_{\text {lin }}}\right) \approx F_{\mathrm{sat}} \ln (1+\Delta R) \approx F_{\mathrm{sat}} \Delta R .
\end{aligned}
$$


Using (A6), we, therefore, have for a QW SESAM

$$
F_{\text {sat }} \Delta R=h \nu N_{0,2 \mathrm{D}} .
$$

Therefore, for samples with the same absorber section the product $F_{\text {sat }} \cdot \Delta R$ remains constant.

\section{APPENDIX II}

\section{APPROXIMATE EXPRESSION OF THE ENERGY DEPOSITED ON THE STRUCTURE DUE TO ISA}

At any given incident fluence $F$, we can calculate the fraction of this incident fluence absorbed by the sample

$$
F_{\mathrm{abs}}=F(1-R(F)) .
$$

As an expression for $R(F)$, we can use the following function:

$$
R(F)=R_{\mathrm{ns}} \frac{\ln \left(1+\frac{R_{\mathrm{lin}}}{R_{\mathrm{ns}}}\left(e^{\frac{F}{F_{\mathrm{sat}}}}-1\right)\right)}{\frac{F}{F_{\mathrm{sat}}}} e^{-\frac{F}{F_{2}}} .
$$

If we consider that we are strongly saturated $\left(F>>F_{\text {sat }}\right)$ and are close to the start of the rollover $\left(F<<F_{2}\right)$, we can approximate this reflectivity function by

$$
R(F)=R_{\mathrm{ns}}\left(1-\frac{F}{F_{2}}\right) .
$$

Therefore, the absorbed energy per area becomes

$$
F_{\mathrm{abs}}=F\left(1-R_{\mathrm{ns}}\left(1-\frac{F}{F_{2}}\right)\right) .
$$

For all samples that we have considered in this study, the nonsaturable losses $1-R_{\mathrm{ns}}$ are negligible; therefore, the expression becomes

$$
F_{\mathrm{abs}}=R_{\mathrm{ns}} \frac{F^{2}}{F_{2}} .
$$

If we consider that damage occurs at a given amount of energy deposited in the sample, we have a condition for the incident damage fluence $F_{\mathrm{d}}$ at which damage occurs

$$
R_{\mathrm{ns}} \frac{F_{\mathrm{d}}^{2}}{F_{2}}=\text { constant. }
$$

Therefore, $F_{\mathrm{d}}$ should scale proportionally to $\sqrt{F_{2}}$.

\section{ACKNOWLEDGMENT}

The authors would like to thank Dr. K. Weingarten for helpful discussions on long-term SESAM damage testing.

\section{REFERENCES}

[1] U. Keller, "Recent developments in compact ultrafast lasers," Nature, vol. 424, pp. 831-838, Aug. 2003.

[2] U. Keller, "Ultrafast solid-state laser oscillators: A success story for the last 20 years with no end in sight," Appl. Phys. B, vol. 100, pp. 15-28, 2010.

[3] U. Keller, K. J. Weingarten, F. X. Kärtner, D. Kopf, B. Braun, I. D. Jung, R. Fluck, C. Hönninger, N. Matuschek, and J. Aus der Au, "Semiconductor saturable absorber mirrors (SESAMs) for femtosecond to nanosecond pulse generation in solid-state lasers," IEEE J. Sel. Top. Quantum Electron., vol. 2, no. 3, pp. 435-453, Sep. 1996.
[4] U. Keller, D. A. B. Miller, G. D. Boyd, T. H. Chiu, J. F. Ferguson, and M. T. Asom, "Solid-state low-loss intracavity saturable absorber for Nd:YLF lasers: An antiresonant semiconductor Fabry-Perot saturable absorber," Opt. Lett., vol. 17, pp. 505-507, 1992.

[5] C. Hönninger, R. Paschotta, F. Morier-Genoud, M. Moser, and U. Keller, "Q-switching stability limits of continuous-wave passive mode locking," J. Opt. Soc. Amer. B, vol. 16, pp. 46-56, Jan. 1999.

[6] C. R. E. Baer, C. Kränkel, C. J. Saraceno, O. H. Heckl, M. Golling, R Peters, K. Petermann, T. Südmeyer, G. Huber, and U. Keller, "Femtosecond thin disk laser with $141 \mathrm{~W}$ of average power," Opt. Lett., vol. 35 , pp. 2302-2304, 2010.

[7] J. Neuhaus, D. Bauer, J. Zhang, A. Killi, J. Kleinbauer, M. Kumkar, S. Weiler, M. Guina, D. H. Sutter, and T. Dekorsy, "Subpicosecond thin-disk laser oscillator with pulse energies of up to 25.9 microjoules by use of an active multipass geometry," Opt. Exp., vol. 16, pp. 20530-20539, 2008.

[8] T. Südmeyer, S. V. Marchese, S. Hashimoto, C. R. E. Baer, G. Gingras, B. Witzel, and U. Keller, "Femtosecond laser oscillators for high-field science," Nature Photon., vol. 2, pp. 599-604, 2008.

[9] A. McPherson, G. Gibson, H. Jara, U. Johann, T. S. Luk, I. A. McIntyre, K. Boyer, and C. K. Rhodes, "Studies of multiphoton production of vacuumultraviolet radiation in the rare gases," J. Opt. Soc. Amer. B, vol. 4, pp. 595-601, Apr. 1987.

[10] M. Ferray, A. L'Huillier, X. F. Li, L. A. Lompr. Mainfray, and C. Manus, "Multiple-harmonic conversion of $1064 \mathrm{~nm}$ radiation in rare gases," $J$. Phys. B: At. Mol. Opt. Phys., vol. 21, pp. L31-L35, 1988.

[11] U. Keller, "Femtosecond to attosecond optics," IEEE Photon. J., vol. 2, pp. 225-228, Apr. 2010.

[12] O. H. Heckl, C. R. E. Baer, C. Kränkel, S. V. Marchese, F. Schapper, M. Holler, T. Südmeyer, J. S. Robinson, J. W. G. Tisch, F. Couny, P. Light, F. Benabid, and U. Keller, "High harmonic generation in a gas-filled hollowcore photonic crystal fiber," Appl. Phys. B, vol. 97, pp. 369-373, 2009.

[13] A. Giesen and J. Speiser, "Fifteen years of work on thin-disk lasers: Results and scaling laws," IEEE J. Sel. Top. Quantum Electron., vol. 13, no. 3, pp. 598-609, May/Jun. 2007.

[14] A. Giesen, H. Hügel, A. Voss, K. Wittig, U. Brauch, and H. Opower, "Scalable concept for diode-pumped high-power solid-state lasers," Appl. Phys. B, vol. 58, pp. 365-372, 1994.

[15] S. V. Marchese, C. R. E. Baer, A. G. Engqvist, S. Hashimoto, D. J. H. C. Maas, M. Golling, T. Südmeyer, and U. Keller, "Femtosecond thin disk laser oscillator with pulse energy beyond the 10-microjoule level," Opt. Exp., vol. 16, pp. 6397-6407, 2008.

[16] V. Magni, "Multielement stable resonators containing a variable lens," $J$. Opt. Soc. Amer. A, vol. 4, pp. 1962-1969, 1987.

[17] G. J. Spühler, K. J. Weingarten, R. Grange, L. Krainer, M. Haiml, V. Liverini, M. Golling, S. Schon, and U. Keller, "Semiconductor saturable absorber mirror structures with low saturation fluence," Appl. Phys. B, vol. 81, pp. 27-32, 2005.

[18] D. J. H. C. Maas, A. R. Bellancourt, M. Hoffmann, B. Rudin, Y. Barbarin, M. Golling, T. Südmeyer, and U. Keller, "Growth parameter optimization for fast quantum dot SESAMs," Opt. Exp., vol. 16, pp. 18646-18656, 2008.

[19] A. E. H. Oehler, M. C. Stumpf, S. Pekarek, T. Südmeyer, K. J. Weingarten, and U. Keller, "Picosecond diode-pumped $1.5 \mu \mathrm{m} \mathrm{Er,Yb:glass} \mathrm{lasers} \mathrm{oper-}$ ating at $10-100 \mathrm{GHz}$ repetition rate," Appl. Phys. B: Lasers Opt., vol. 99 , pp. 53-62, 2010

[20] U. Keller and A. C. Tropper, "Passively modelocked surface-emitting semiconductor lasers," Phys. Rep., vol. 429, pp. 67-120, Jun. 2006.

[21] L. Krainer, R. Paschotta, S. Lecomte, M. Moser, K. J. Weingarten, and U. Keller, "Compact Nd: $\mathrm{YVO}_{4}$ lasers with pulse repetition rates up to 160 GHz," IEEE J. Quantum Electron., vol. 38, no. 10, pp. 1331-1338, Oct. 2002.

[22] A. E. H. Oehler, T. Südmeyer, K. J. Weingarten, and U. Keller, "100 GHz passively mode-locked Er:Yb:glass laser at $1.5 \mu \mathrm{m}$ with 1.6-ps pulses," Opt. Exp., vol. 16, pp. 21930-21935, Dec. 2008.

[23] D. J. H. C. Maas, A.-R. Bellancourt, B. Rudin, M. Golling, H. J. Unold, T. Südmeyer, and U. Keller, "Vertical integration of ultrafast semiconductor lasers," Appl. Phys. B, vol. 88, pp. 493-497, 2007.

[24] R. Paschotta and U. Keller, "Passive mode locking with slow saturable absorbers," Appl. Phys. B, vol. 73, pp. 653-662, 2001.

[25] R. Grange, M. Haiml, R. Paschotta, G. J. Spuhler, L. Krainer, M. Golling, O. Ostinelli, and U. Keller, "New regime of inverse saturable absorption for self-stabilizing passively mode-locked lasers," Appl. Phys. B, vol. 80, pp. 151-158, 2005.

[26] J. Aus der Au, D. Kopf, F. Morier-Genoud, M. Moser, and U. Keller, "60-fs pulses from a diode-pumped Nd:glass laser," Opt. Lett., vol. 22, pp. 307-309, 1997. 
[27] E. Innerhofer, T. Südmeyer, F. Brunner, R. Häring, A. Aschwanden, R. Paschotta, U. Keller, C. Hönninger, and M. Kumkar, "60 W average power in 810-fs pulses from a thin-disk Yb:YAG laser," Opt. Lett., vol. 28 , pp. 367-369, 2003

[28] M. Haiml, R. Grange, and U. Keller, "Optical characterization of semiconductor saturable absorbers," Appl. Phys. B, vol. 79, pp. 331-339, Aug. 2004.

[29] D. J. H. C. Maas, B. Rudin, A.-R. Bellancourt, D. Iwaniuk, S. V. Marchese, T. Südmeyer, and U. Keller, "High precision optical characterization of semiconductor saturable absorber mirrors," Opt. Exp., vol. 16, pp. 75717579,2008

[30] I. D. Jung, F. X. Kärtner, L. R. Brovelli, M. Kamp, and U. Keller, "Experimental verification of soliton modelocking using only a slow saturable absorber," Opt. Lett., vol. 20, pp. 1892-1894, 1995.

[31] F. X. Kärtner, I. D. Jung, and U. Keller, "Soliton mode-locking with saturable absorbers," IEEE J. Sel. Top. Quant., vol. 2, pp. 540-556, Sep. 1996.

[32] P. Langlois, M. Joschko, E. R. Thoen, E. M. Koontz, F. X. Kärtner, E. P. Ippen, and L. A. Kolodziejski, "High fluence ultrafast dynamics of semiconductor saturable absorber mirrors," Appl. Phys. Lett., vol. 75, pp. 3841-3843, 1999.

[33] T. R. Schibli, E. R. Thoen, F. X. Kärtner, and E. P. Ippen, "Suppression of Q-switched mode locking and break-up into multiple pulses by inverse saturable absorption," Appl. Phys. B, vol. 70, pp. S41-S49, 2000.

[34] L. R. Brovelli, U. Keller, and T. H. Chiu, "Design and Operation of antiresonant Fabry-Perot saturable semiconductor absorbers for modelocked solid-state lasers," J. Opt. Soc. Amer. B, vol. 12, pp. 311-322, Feb. 1995.

[35] M. Haiml, U. Siegner, F. Morier-Genoud, U. Keller, M. Luysberg, R. C. Lutz, P. Specht, and E. R. Weber, "Optical nonlinearity in low-temperaturegrown GaAs: Microscopic limitations and optimization strategies," Appl. Phys. Lett., vol. 74, pp. 3134-3136, May 24, 1999.

[36] E. W. Van Stryland, M. A. Woodall, H. Vanherzeele, and M. J. Soileau, "Energy band-gap dependence of two-photon absorption," Opt. Lett., vol. 10, pp. 490-492, Oct. 1985

[37] C. R. E. Baer, C. Kränkel, C. J. Saraceno, O. H. Heckl, M. Golling, T. Südmeyer, R. Peters, K. Petermann, G. Huber, and U. Keller, "Femtosecond $\mathrm{Yb}: \mathrm{Lu}_{2} \mathrm{O}_{3}$ thin disk laser with $63 \mathrm{~W}$ of average power," Opt. Lett., vol. 34, pp. 2823-2825, 2009.

[38] K. Weingarten, "private communication," Time Bandwidth Products, Zurich, Switzerland, 2010.

[39] K. H. Fong, K. Kikuchi, C. S. Goh, S. Y. Set, R. Grange, M. Haiml, A. Schlatter, and U. Keller, "Solid-state Er:Yb:glass laser mode-locked by using single-wall carbon nanotube thin film," Opt. Lett., vol. 32, pp. 38 40, 2007.

[40] Y. C. Chen, Y. C. Chen, N. R. Raravikar, L. S. Schadler, P. M. Ajayan, Y. P. Zhao, T. M. Lu, G. C. Wang, and X. C. Zhang, "Ultrafast optical switching properties of single-wall carbon nanotube polymer composites at $1.55 \mu \mathrm{m}, "$ Appl. Phys. Lett., vol. 81, pp. 975-977, Aug 5, 2002.

[41] J. H. Yim, W. B. Cho, S. Lee, Y. H. Ahn, K. Kim, H. Lim, G. Steinmeyer, V. Petrov, U. Griebner, and F. Rotermund, "Fabrication and characterization of ultrafast carbon nanotube saturable absorbers for solid-state laser mode locking near $1 \mu \mathrm{m}$," Appl. Phys. Lett., vol. 93, Oct. 20, 2008.

[42] S. Kivisto, T. Hakulinen, A. Kaskela, B. Aitchison, D. P. Brown, A. G. Nasibulin, E. I. Kauppinen, A. Harkonen, and O. G. Okhotnikov, "Carbon nanotube films for ultrafast broadband technology," Opt. Exp., vol. 17, pp. 2358-2363, Feb. 16, 2009.

[43] D. Maas, MIXSELs-A new class of ultrafast semiconductor lasers Dissertation at ETH Zurich, Nr. 18121, Hartung-Gorre Verlag, Konstanz, 2009.

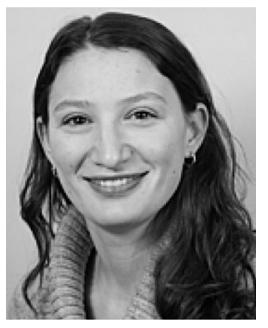

Clara J. Saraceno was born in Buenos Aires, Argentina, on September 13, 1983. She received the Dipl.Ing. and M.S. degrees from the Insititut d'Optique Graduate School, Palaiseau, France, in September 2007. From September 2007 to September 2008, she also received training in the R\&D Department of the Ultrafast Division (RLS), Coherent, Inc., Santa Clara, CA, where she developed interest for ultrafast laser physics. She is currently working toward the Ph.D. degree in Prof. Ursula Keller's group (U1trafast Laser Physics) at Eidgenössische Technische Hochschule (ETH), Zurich, Switzerland.

Her current research interests include high-power ultrafast mode locked oscillators and their application to drive high field physics experiments.

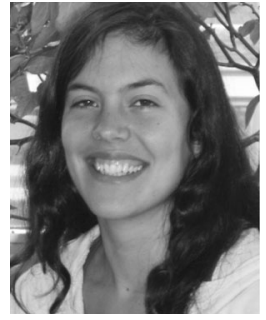

Cinia Schriber was born in Mexico City, on April 28, 1985. She received the B.S. degree in general physics in 2009 from Eidgenössische Technische Hochschule (ETH), Zurich, Switzerland, where she is currently working toward the M.S. degree in general physics.

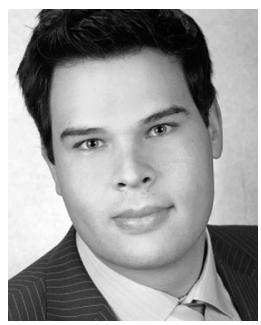

Mario Mangold was born in Landshut, Germany, on November 7, 1986. He received the B.S. degree in general physics in 2009 from Ludwig Maximilian University, Munich, Germany. He is currently working toward the M.S. degree in general physics at the Eidgenössische Technische Hochschule (ETH), Zurich, Switzerland.

His current research interests include the characterization of VECSEL gain materials.

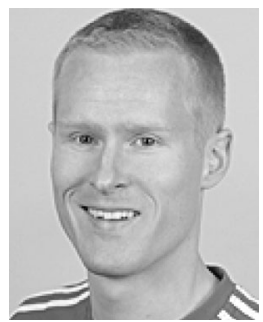

Martin Hoffmann was born in Dessau, Germany, on October 15, 1979. He received the Diploma degree in physics in 2007 from the Federal Institute of Technology, Zurich, Switzerland, where he is currently working toward the Ph.D. degree in Prof. Ursula Keller's group (Ultrafast Laser Physics) at Eidgenössische Technische Hochschule (ETH), Zurich.

His current research interests include semiconductor saturable absorber mirrors mode-locked quantum dot and quantum well-based vertical external cavity surface emitting lasers, both optically and electrically

pumped.

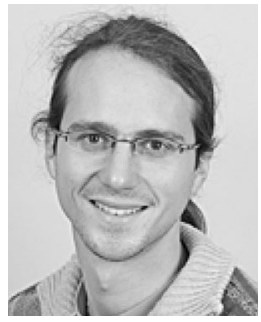

Oliver H. Heckl was born in Augsburg, Germany, on December 2, 1981. He received the Diploma degree in physics from the University of Augsburg, Augsburg, in 2007. He is currently working toward the Ph.D. degree in Prof. Ursula Keller's Group (Ultrafast Laser Physics) at Eidgenössische Technische Hochschule (ETH), Zurich, Switzerland.

His current research interests include high peakand average-power mode-locked thin-disk lasers and their application in oscillator-driven high field experiments.

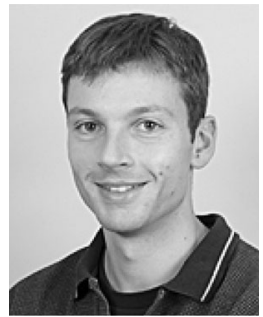

Cyrill R. E. Baer was born in Herisau, Switzerland, on January 18,1981 . He received the M.S. degree in physics from the University of Basel, Switzerland, in 2006. He is currently working toward the Ph.D. degree in Prof. Ursula Keller's Group (Ultrafast Laser Physics) at Eidgenössische Technische Hochschule (ETH), Zurich, Switzerland.

His current research interests include the investigation of new materials for high-power ultrafast laser oscillators, focusing on the generation of short pulses, high pulse energies, and their application in high field

physics experiments.

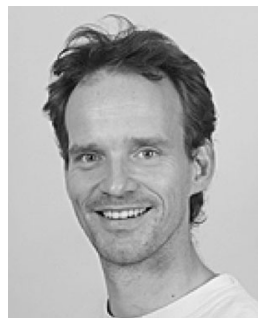

Matthias Golling was born in Sulz, Germany, on May 20, 1971. He received the Diploma degree in electrical engineering and the Ph.D. degree in engineering both from the University of Ulm, Ulm, Germany, in 1997 and 2004, respectively.

$\mathrm{He}$ is currently the Leader of the molecular beam epitaxy (MBE) activities at the Ultrafast Laser Physics Group, Eidgenössische Technische Hochschule (ETH), Zurich, Switzerland. His current research interests include the design and epitaxy of lasers and saturable absorbers. 


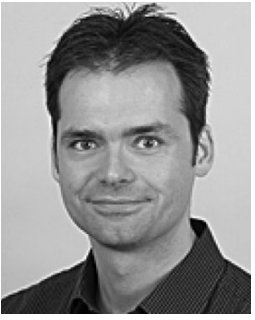

Thomas Südmeyer received the Diploma degree in physics from the University of Hanover, Hanover, Germany, and the Ph.D. degree from Eidgenössische Technische Hochschule (ETH), Zurich, Switzerland, in 1998 and 2003, respectively. His Ph.D. research at ETH was focused on the first mode-locked thindisk lasers and the realization of novel nonlinear systems.

In 1999, during a six-month EU research fellowship at the Institute of Photonics, Glasgow, U.K., he became interested in high-power mode-locked lasers. From 2003 to 2005, he developed industrial laser solutions such as high-power 266-nm lasers in the Department of Photonics Research, Sony Corporation, Tokyo, Japan. Since 2005, he has been Head of the ultrafast laser section in Ursula Keller's group, ETH. He is the author of more than 40 papers in international peer-reviewed journals, two book chapters, and he holds or applied for six patents. He has been the Coordinator of the FP6 European Project MULTIWAVE. He is an Associate Editor for Optics Express, and serves the optical community in various conference committees. His current scientific interests include femtosecond diode-pumped solid-state lasers, multigigahertz repetition rate lasers, mode-locked semiconductor lasers, and their applications in nonlinear optics, metrology, communication, and high field science.

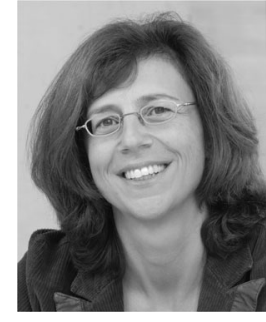

Ursula Keller received the Diploma degree in physics from Eidgenössische Technische Hochschule (ETH), Zurich, Switzerland, and the Ph.D. degree in applied physics from Stanford University, Stanford, CA, in 1984 and1989, respectively.

In 1993, she joined ETH as a Professor of physics. From 1989 to 1993, she was a member of technical staff at AT\&T Bell Laboratories, New Jersey. She has published more than 300 peer-reviewed journal papers and 11 book chapters, and she holds or applied for 17 patents. Her research interests include exploring and pushing the frontiers in ultrafast science and technology: ultrafast solid-state and semiconductor lasers, frequency comb generation and stabilization, attosecond pulse generation, and science using high harmonic generation.

Dr. Keller was the recipient of OSA Fraunhofer/Burley Prize in 2008, the Philip Morris Research Award in 2005, the First-Placed Award of the Berthold Leibinger Innovation Prize in 2004, and the Carl Zeiss Research Award in 1998. She is an OSA Fellow and an Elected Foreign Member of the Royal Swedish Academy of Sciences and the German Academy Leopoldina. 